OPEN ACCESS

Check for updates

FAST TRACK

\title{
Incidence, clinical outcomes, and transmission dynamics of severe coronavirus disease 2019 in California and Washington: prospective cohort study
}

\author{
Joseph A Lewnard, ${ }^{1,2,3}$ Vincent X Liu, ${ }^{4}$ Michael L Jackson, ${ }^{5}$ Mark A Schmidt, ${ }^{6}$ Britta L Jewell,,${ }^{7,8}$ \\ Jean P Flores, ${ }^{9}$ Chris Jentz, ${ }^{9}$ Graham R Northrup, ${ }^{3}$ Ayesha Mahmud, ${ }^{10}$ Arthur L Reingold, ${ }^{1}$ \\ Maya Petersen, ${ }^{1}$ Nicholas P Jewell, ${ }^{1,11}$ Scott Young, ${ }^{9,12}$ Jim Bellows ${ }^{9}$
}

For numbered affiliations see end of the article.

Correspondence to: I A Lewnard jlewnard@berkeley.edu

(or@jLewnard on Twitter

ORCID 0000-0002-8505-8839)

Additional material is published online only. To view please visit the journal online.

Cite this as: $B M J$ 2020;369:m1923 http://dx.doi.org/10.1136/bmj.m1923

Accepted: 12 May 2020

\section{ABSTRACT}

OBJECTIVE

To understand the epidemiology and burden of severe coronavirus disease 2019 (covid-19) during the first epidemic wave on the west coast of the United States.

DESIGN

Prospective cohort study.

\section{SETTING}

Kaiser Permanente integrated healthcare delivery systems serving populations in northern California, southern California, and Washington state.

\section{PARTICIPANTS}

1840 people with a first acute hospital admission for confirmed covid-19 by 22 April 2020, among 9596321 healthcare plan enrollees. Analyses of hospital length of stay and clinical outcomes included 1328 people admitted by 9 April 2020 (534 in northern California, 711 in southern California, and 83 in Washington).

\section{MAIN OUTCOME MEASURES}

Cumulative incidence of first acute hospital admission for confirmed covid-19, and subsequent probabilities of admission to an intensive care unit (ICU) and mortality, as well as duration of hospital stay and ICU stay. The effective reproduction number $\left(R_{E}\right)$ describing transmission dynamics was estimated for each region.

RESULTS

As of 22 April 2020, cumulative incidences of a first acute hospital admission for covid-19 were 15.6 per

\section{WHAT IS ALREADY KNOWN ON THIS TOPIC}

Coronavirus disease 2019 (covid-19) has overwhelmed the healthcare systems of certain countries and regions, with major proportions of patients requiring critical care

The west coast of the United States, however, has been spared severe healthcare disruption associated with surges in patients with severe covid-19 seen in other regions

\section{WHAT THIS STUDY ADDS}

In enrollees of the Kaiser Permanente healthcare service plan resident in California and Washington state, between 15 and 23 people were admitted to hospital for covid-19 per 100000 cohort members

About one half of male patients and one third of female patients in this study who were admitted to hospital required critical care, and $23 \%$ of male patients and $15 \%$ of female patients died following admission

Transmission intensity declined in March 2020 in conjunction with implementation of interventions aiming to mitigate transmission
100000 cohort members in northern California, 23.3 per 100000 in southern California, and 14.7 per 100000 in Washington. Accounting for censoring of incomplete hospital stays among those admitted by 9 April 2020, the estimated median duration of stay among survivors was 9.3 days (with $95 \%$ staying 0.8 to 32.9 days) and among non-survivors was 12.7 days (1.6 to 37.7 days). The censoring adjusted probability of ICU admission for male patients was $48.5 \%$ (95\% confidence interval $41.8 \%$ to $56.3 \%$ ) and for female patients was $32.0 \%$ (26.6\% to $38.4 \%$ ). For patients requiring critical care, the median duration of ICU stay was 10.6 days (with $95 \%$ staying 1.3 to 30.8 days). The censoring adjusted case fatality ratio was $23.5 \%$ (95\% confidence interval $19.6 \%$ to $28.2 \%$ ) among male inpatients and $14.9 \%$ (11.8\% to $18.6 \%$ ) among female inpatients; mortality risk increased with age for both male and female patients. Reductions in $R_{E}$ were identified over the study period within each region.

CONCLUSIONS

Among residents of California and Washington state enrolled in Kaiser Permanente healthcare plans who were admitted to hospital with covid-19, the probabilities of ICU admission, of long hospital stay, and of mortality were identified to be high. Incidence rates of new hospital admissions have stabilized or declined in conjunction with implementation of social distancing interventions.

\section{Introduction}

Months after its emergence, the novel severe acute respiratory syndrome coronavirus 2 (SARS-CoV-2), responsible for coronavirus disease 2019 (covid-19), has resulted in a pandemic, with cases reported in nearly every country. ${ }^{1}$ In total, 1364061 cases and 82246 deaths were reported in the United States as of 14 May 2020 , representing the greatest sum of any country. ${ }^{2}$ Surges in covid-19 cases have overwhelmed the capacity of hospital and healthcare systems in regions of the US, mirroring the considerable disruption witnessed in other countries. ${ }^{3-5}$ However, the epidemiology of covid-19 in the US remains poorly described, including clinical variables of disease progression, the risk of admission to an intensive care unit (ICU), and death by patient age and sex, and the duration of hospital stay. As such, efforts to forecast trajectories of the epidemic to guide planning and response in the US and other high income settings have relied almost entirely on data from China to inform these variables, ${ }^{6}$ which might not adequately reflect clinical circumstances elsewhere. 
Metropolitan areas of the western US were among the first to report covid-19 importations and domestically acquired cases. Washington state announced the first known imported case of covid-19 in the US on 21 January 2020. Community transmission of SARS-CoV-2 has occurred at least since February in northern California, leading to covid-19 related deaths as early as 6 February. Most of the cases in California are concentrated in counties surrounding the San Francisco Bay in northern California, and Los Angeles in southern California. These regions were also among the first to implement intensive public health interventions aiming to curtail transmission. Statewide emergency declarations came into effect on 29 February in Washington and 4 March in California. Social distancing recommendations for vulnerable populations were issued in San Francisco on 6 March and large gatherings were banned in Washington on 11 March and in Los Angeles on 12 March. Large scale stay at home orders were implemented on 17 March for the six counties of the San Francisco Bay area, and statewide in California on 19 March and in Washington on 24 March. Understanding the impact of these interventions is of crucial importance to inform their broader use, and could help to account for emerging regional differences in the severity of covid-19 outbreaks across the country. ${ }^{7}$

To inform the epidemiology of covid-19 in these regions, we analyzed healthcare data for all people admitted to hospital with covid-19 within the cohort of 9596321 who received comprehensive, integrated care from Kaiser Permanente healthcare systems in northern California, southern California, and Washington state.

\section{Methods \\ Setting}

The Kaiser Permanente healthcare systems for northern California, southern California, and Washington state deliver fully integrated healthcare to diverse membership cohorts. Profiles of enrollees by age, race, and sex are similar to those of the general populations of the states of California and Washington (see supplementary table S1). As most enrollees receive employer sponsored health insurance coverage, however, fewer Kaiser Permanente plan members either have low incomes or reside in low income census tracts compared with national or regional averages. ${ }^{8-11}$ Compared with $33 \%$ of adults in the US general population, $41 \%$ of Kaiser Permanente enrollees in northern California and 31\% in southern California aged 25 years or older are estimated to have completed four or more years of college education; similar estimates are not yet available for enrollees in Washington as membership was only established in 2017..$^{1213}$

\section{Study design}

We analyzed clinical and administrative data captured from all Kaiser Permanente members who had been admitted to hospital within the northern California, southern California, and Washington healthcare delivery systems with covid-19 laboratory or clinical diagnoses at any recorded healthcare encounter by end of day on 22 April 2020; for analyses of patient outcomes, we included those admitted by 9 April 2020, because data for at least 14 days' follow-up were available at time of analysis. We considered patients to have clinically confirmed covid-19 if no positive test result was available and diagnosis codes included any of the diagnoses listed in supplementary table S2. Clinical diagnoses were overruled by negative laboratory test results.

Recorded encounters included hospital, outpatient, and telehealth visits as well as uses of laboratory diagnostic services. We limited hospital admissions to acute inpatient admissions occurring between 14 days before and 28 days after the first encounter when a patient was flagged for exposure to SARS-CoV-2, was designated as a "person under investigation" for covid-19, or had confirmed covid-19. Because only the most recent designation as exposure to SARS-Cov-2, person under investigation, or confirmed covid-19 was available for the Washington cohort, we considered hospital admissions for covid-19 to include those for which admission or discharge occurred within 30 days of the encounter with this most recent status assigned. Observational admissions were excluded. For both Californian cohorts, we sourced hospital admission events from electronic medical records and outside medical claims. Hospital admissions in the Washington cohort were sourced from a centralized admissions database.

Patients discharged at the end of their most recent hospital stay were considered to be survivors if they had not died by end of day 22 April; while standardized discharge criteria were not in place, considerations included clinical recovery (no hypoxia or mild hypoxia that corrects easily with supplemental oxygen), low perceived risk of clinical deterioration, and access to appropriate post-discharge clinical care at the discharge location, consistent with Centers for Disease Control and Prevention guidance. ${ }^{1415}$ We used competing risk parametric survival methods to account for censoring of observations from currently admitted patients for all analyses. Available data for patients included dates of covid-19 clinical encounters, age, sex, dates of hospital stay, total duration of hospital stay, duration of ICU stay, survival or non-survival outcome (for completed hospital admissions only), and covid-19 diagnostic tests performed in any setting, along with the results.

\section{Statistical analysis}

\section{Incidence estimation}

For each of the three cohorts we estimated daily and cumulative incidences of covid-19 related hospital admissions within the full cohort population and within 10 year age stratums (ages 0-9, 10-19, 20-29, 30-39, 40-49, 50-59, 60-69, 70-79, and $\geq 80$ years). Hospital admission case data included all patients admitted by end of day 22 April. 


\section{Duration of hospital stay}

We aimed to infer distributions of the duration of hospital stay among all patients admitted by 9 April 2020, and the distribution of the duration of ICU stay among patients receiving intensive care. We computed length of stay as the number of unique calendar days during which participants were in hospital; durations were summed across all hospital stays for readmitted patients. For these analyses, we used the CFC package ${ }^{16}$ in $\mathrm{R}$ (version 1.1.463; R Foundation for Statistical Computing, Vienna, Austria) to fit age adjusted Weibull competing risk models. Outcomes included discharge (alive), mortality, or right censoring (for ongoing hospital admission).

Duration of ICU stay was available only among participants with completed hospital admissions. For unbiased inference of the duration of ICU stay, we resampled observations from participants' conditional distribution of ICU lengths of stay, given their duration of hospital admission, age group, and survivor or non-survivor status. We defined sampling weights according to the (unconditional) distribution of total durations for hospital admissions across these patient stratums. We fitted Weibull distribution parameters to the resampled data through maximum likelihood to reconstruct unbiased distributions of the total duration of ICU stay.

\section{Probability of ICU admission and mortality}

We used a similar approach to correct for censoring of recent hospital admissions when estimating age and sex specific probabilities of ICU admission and death among hospital inpatients admitted by 9 April 2020 . Using generalized linear models with a Poisson link function, we estimated the conditional probability of each outcome among those with completed hospital admissions given the duration of hospital stay, age, and sex, accounting for all two way interactions among these covariates needed to minimize values of the bayesian information criterion. We then integrated the estimated conditional probabilities of each outcome over all durations of hospital admissions, weighted by the probability distribution of durations for hospital admissions within each age and sex stratum.

\section{Transmission dynamics}

We used hospital admission data to estimate time varying values of the effective reproduction number $\left(R_{E}\right)$, which describes the number of secondary infections resulting from infections acquired on a given day. ${ }^{17}$ We compared $R_{E}$ estimates generated under eight analyses defined by alternative parameterizations of the incubation period, the time from symptoms onset to hospital admission, and the infection to hospital admission ratio, each informed by data from distinct studies. $^{18-28}$

We sampled the date of SARS-CoV-2 infection for each patient admitted to hospital, assuming time from infection to hospital admission was distributed according to the sum of random draws from fitted distributions of the time from infection to symptoms onset (incubation period) and the time from symptoms onset to hospital admission. We considered two parameterizations of the incubation period: first, we inferred duration by sampling from previous parameterizations of the incubation period duration on the basis of independent data sources from multiple countries ${ }^{18 \text { 19-21 }}$ (weighted by the number of participants in each of these studies) and by fitting a Weibull distribution to the resulting pooled sample through maximum likelihood. Second, we considered a log normal parameterization of the incubation period fitted in a previous study. ${ }^{20}$

We also used estimates from two different studies for the time from symptoms onset to hospital admission. The first study reported variables of a Gamma distribution providing the best fit to patient level observations (compared with Weibull and $\log$ normal parameterizations). ${ }^{22}$ For the second study, we fitted Gamma, Weibull, and log normal distribution parameters against the reported mean and interquartile range of time from symptoms onset to hospital admission, finding that a Gamma distribution minimized summed squared errors compared with reported values. ${ }^{23}$ To account for right censoring of infections in those who were not admitted to hospital by the end of the study period, we divided the number of observed infections in those admitted to hospital that were estimated to have been acquired each date by the proportion of those with infections who would be expected to experience hospital admission by 22 April, according to the cumulative distribution function of time from infection to hospital admission.

We weighted the number of infections occurring for each observed patient admitted to hospital by previous estimates of the age specific probability of hospital admission, given infection, from two distinct studies. $^{24} 25$ We propagated uncertainty in this probability by fitting log normal distributions to reported empirical means and 95\% uncertainty bounds within each age group (aggregated at ages 0-19 years), for each study, and taking independent draws from these fitted distributions for analyses. Efforts to parameterize the proportion of infections necessitating hospital admission under the assumption of Beta distributed probabilities resulted in greater summed squared errors compared with reported means and 95\% uncertainty bounds.

To estimate $R_{E}$ for new infections acquired each day in each cohort, we applied the method of Wallinga and Teunis $^{17}$ to our reconstructed estimates of daily new infections, using the distribution of the serial interval to assign the probability of a transmissionlinkbetween any two infections on differing days. Similar to the analysis described for incubation periods, we sampled from serial interval estimates from previous studies ${ }^{18} 26-28$ according to the number of participants for whom data were available, and fitted a Weibull distribution to the sampled data by maximum likelihood. To correct for right censoring of transmission pairs, we divided estimates of $R_{E}$ for infections acquired each day $t$ by the cumulative distribution function of the serial interval 
evaluated over the period from day $t$ to the end of the study.

\section{Patient and public involvement}

As the study involved secondary analysis of data, patients and members of the public were not directly involved in the study design.

\section{Results}

Cumulatively, 1840 members were admitted to hospital with a confirmed diagnosis of covid-19 as of 22 April 2020: 678 in the northern California cohort, 1059 in the southern California cohort, and 103 in the Washington cohort (see supplementary table S3). Median time from admission to covid-19 diagnosis declined from three days to zero days between February and April 2020, and an increasing proportion of admitted patients received a covid-19 diagnosis before admission over this period (see supplementary table S4).

In total, 1328 people were admitted to hospital by 9 April 2020, and thus had 14 days or more of follow-up at time of data analysis for assessment of clinical outcomes (table 1). Among these patients, 534 were from the northern California cohort, 711 from the southern California cohort, and 83 from the Washington cohort. The median age of patients was 61 years (range 18-103 years), with $50 \%$ aged 48 to 72 years. Four $(0.3 \%)$ patients were younger than 20 years, $528(39.8 \%)$ were 65 years or older, and 162 $(12.2 \%)$ were 80 years or older; $741(55.8 \%)$ were male patients. By end of 22 April 2020, laboratory confirmed diagnosis of covid-19 was available for 1271 (95.7\%) patients admitted to hospital by 9 April 2020.

Hospital admissions were complete for 1176 (88.6\%) patients who had been admitted to hospital by 9 April 2020; 1095 of whom had complete data on survival or non-survival status (table 1). Among patients with completed hospital stays and outcomes recorded, 202 (18.4\%) had died by end of day 22 April 2020. Data on ICU admissions were available for 1101 people (only those in both Californian cohorts), among whom 333 were admitted to ICU.

Incidence of covid-19 related hospital admissions For the period ending 22 April 2020, the estimated cumulative incidences of covid-19 related hospital admissions were 15.6 per 100000 cohort members for the northern California cohort, 23.3 per 100000 for the southern California cohort, and 14.7 per 100000 for the Washington cohort (fig 1). Incidence increased with age, with hospital admissions by 22 April 2020 in those aged 80 or older reaching 74.0 per 100000 members in the northern California cohort, 90.4 per 100000 in the southern California cohort, and 46.7 in the Washington cohort (fig 2). Daily rates of hospital admissions reached their highest levels on 23 March in the northern California cohort, 1 April in the southern California cohort, and 4 April in the Washington cohort, with new daily admissions of $0.6,1.0$, and 1.0 per 100000 cohort members, respectively.

\section{Duration of hospital stay}

Accounting for censoring (see supplementary table S5), the estimated median length of stay for all patients admitted to hospital by 9 April 2020 was 10.1 days, with $50 \%$ of hospital stays lasting between 5.3 and 16.8 days and 95\% lasting between 0.9 and 34.5 days (fig 3); similarly, the observed median duration of stay for patients admitted by 15 March 2020 was 11 days. Model estimated median durations of stay were 9.3 days for survivors and 12.7 days for non-survivors, with 95\% of these patients staying 0.8 to 32.9 days and 1.6 to 37.7 days, respectively. The estimated median duration of ICU stay was 10.5 days among patients receiving intensive care, with $50 \%$ of ICU stays lasting between 6.0 and 16.4 days and 95\% lasting between 1.3 and 30.7 days. These estimates did not differ in sensitivity analyses restricted to patients with laboratory confirmed covid-19 (see supplementary table S6).

\section{Intensive care requirements and case fatality risk}

Across all age groups and sexes (weighted by their representation among new admissions) and accounting for censoring of recent hospital admissions, the estimated probability of ICU admission was $40.7 \%$ (95\% confidence interval $36.0 \%$ to $46.1 \%$ ) and the probability of death was $18.9 \%$ (16.3\% to $21.9 \%$ ) (fig 4). Risk of death increased with age, reaching $37.3 \%$ (30.6\% to $45.0 \%$ ) among participants aged 80 years or older admitted to hospital. Additionally, male patients were more likely to be admitted to ICU than female patients $(48.5 \%(41.8 \%$ to $56.3 \%) \vee 32.0 \%(26.6 \%$ to $38.4 \%)$ ) and more likely to die $(23.5 \%$ (19.6\% to $28.2 \%) v 14.9 \%(11.8 \%$ to $18.6 \%))$. These results likewise did not differ in sensitivity analyses restricted to those with laboratory confirmed covid-19 (see supplementary table S7).

\section{Transmission dynamics}

Based on all possible combinations of the parameterizations listed in supplementary tables S8 and S9, we estimated that people acquiring infection on 1 March 2020 were expected to be responsible for between 1.39 (95\% confidence interval 1.14 to 1.69) and 1.54 (1.18 to 1.98) secondary infections, on average $\left(R_{E}\right)$, in the Kaiser Permanente northern California region; between 2.06 (1.47 to 2.94) and 2.49 (1.88 to 3.33) in the southern California region; and between 1.31 (0.00 to 2.94) and 1.53 (0.00 to 2.59) in the Washington region (see supplementary fig S2). Wider uncertainty intervals are propagated in $R_{E}$ estimates for Washington owing to the smaller cohort size and lower absolute number of hospital admissions. After implementation of mitigation interventions by state, county, and municipal governments, the estimated $R_{E}$ among those acquiring infection on 1 April 2020 was between 0.81 (0.62 to 1.03 ) and 0.90 (0.68 to 1.16) in the Kaiser Permanente northern California region, between 0.78 (0.68 to 0.90$)$ and 0.87 (0.77 to 0.98$)$ in the southern California region, and between $0.78(0.00$ to 1.51 ) and 0.86 (0.00 to 1.28$)$ in the Washington region. $^{29}$ 


\begin{tabular}{|c|c|c|c|c|}
\hline \multirow[b]{2}{*}{ Characteristics } & \multicolumn{3}{|c|}{ Kaiser Permanente region } & \multirow[b]{2}{*}{$\begin{array}{l}\text { All regions } \\
(n=9596321)^{\star}\end{array}$} \\
\hline & $\begin{array}{l}\text { Northern California } \\
(n=4348020)^{\star}\end{array}$ & $\begin{array}{l}\text { Southern California } \\
(n=4548811)^{\star}\end{array}$ & $\begin{array}{l}\text { Washington } \\
(\mathrm{n}=699490)^{\star}\end{array}$ & \\
\hline No admitted to hospital & 534 & 711 & 83 & 1328 \\
\hline Median (range); interquartile range & $61(19-103) ; 49-73$ & $60(18-100) ; 47-71$ & $62(27-91) ; 52-72$ & $61(18-103) ; 48-72$ \\
\hline \multicolumn{5}{|l|}{ Age group (years): } \\
\hline $0-9$ & $0(0)$ & $0(0)$ & $0(0)$ & $0(0)$ \\
\hline $10-19$ & $1(0.2)$ & $3(0.4)$ & $0(0)$ & $4(0.3)$ \\
\hline $20-29$ & $21(3.9)$ & $26(3.7)$ & $1(1.2)$ & $48(3.6)$ \\
\hline $30-39$ & $38(7.1)$ & $77(10.8)$ & $5(6.0)$ & $120(9.0)$ \\
\hline $40-49$ & $80(15.0)$ & $100(14.1)$ & $13(15.7)$ & $193(14.5)$ \\
\hline $50-59$ & $109(20.5)$ & $144(20.3)$ & $16(19.3)$ & $269(20.3)$ \\
\hline $60-69$ & $118(22.1)$ & $163(22.9)$ & $21(25.3)$ & $302(22.8)$ \\
\hline $70-79$ & $91(17.1)$ & $119(16.7)$ & $19(22.9)$ & $229(17.3)$ \\
\hline$\geq 80$ & $75(14.1)$ & $79(11.1)$ & $8(9.6)$ & $162(12.2)$ \\
\hline Male sex & $301(56.5)$ & $396(55.7)$ & $44(53.0)$ & $741(55.8)$ \\
\hline \multicolumn{5}{|l|}{ Events before 23 April 2020: } \\
\hline Laboratory confirmed covid-19 & $502(94.0)$ & $686(96.5)$ & $83(100)$ & $1271(95.7)$ \\
\hline Hospital admission complete & $480(89.9)$ & $621(87.3)$ & $75(90.4)$ & $1176(88.6)$ \\
\hline Discharge alive $\dagger$ & $393 / 472(83.3)$ & $434 / 548(79.2)$ & $66 / 75(88.0)$ & $893 / 1095(81.6)$ \\
\hline Death $t$ & $79 / 472(16.7)$ & $114 / 548(20.8)$ & $9 / 75(12.0)$ & $202 / 1095(18.4)$ \\
\hline \multicolumn{5}{|l|}{ Intensive care unit admissionł } \\
\hline $\begin{array}{l}\text { Among cases with completed } \\
\text { hospital admissions }\end{array}$ & $134 / 480(27.9)$ & $199 / 621(32.0)$ & - & - \\
\hline Among survivors & $81 / 393(20.6)$ & $115 / 434(26.5)$ & - & - \\
\hline Among non-survivors & $53 / 79(67.1)$ & $84 / 114(73.7)$ & - & - \\
\hline
\end{tabular}

Northern California
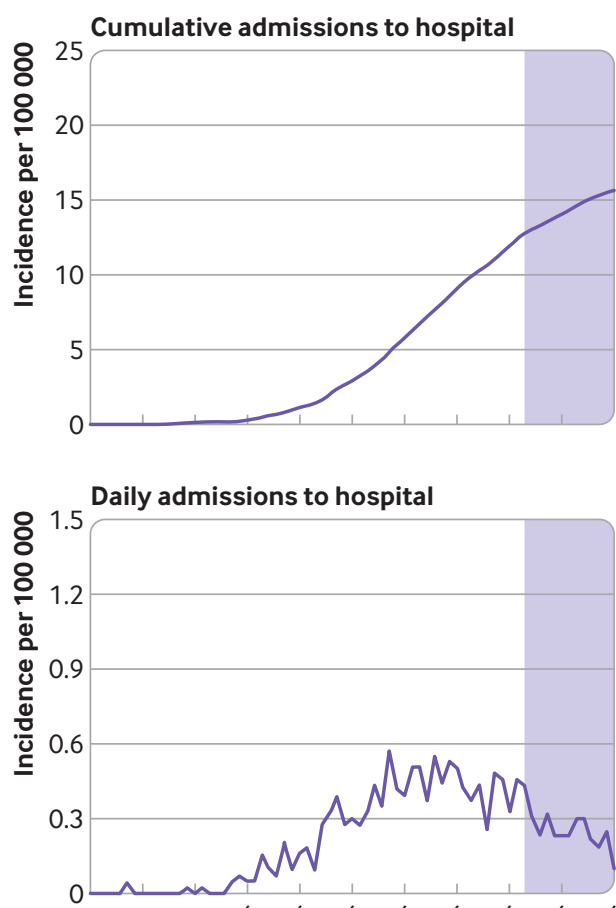

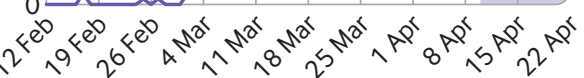

Date of hospital admission


Southern California

Washington state
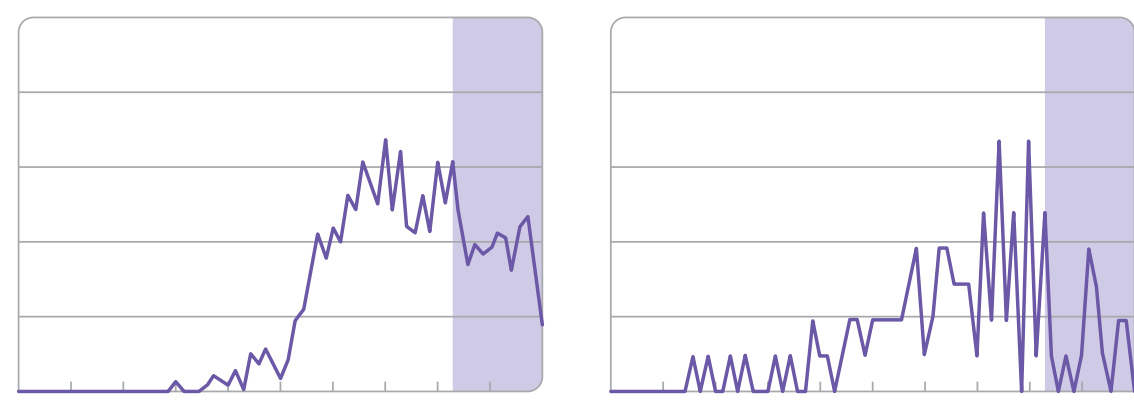

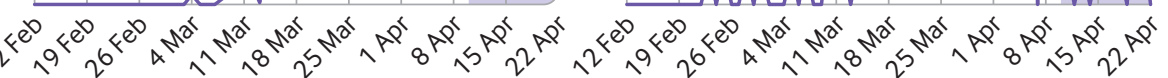

Date of hospital admission

Fig 1 | Cumulative incidence and daily incidence of first acute inpatient admissions in 2020 with confirmed coronavirus disease 2019 among participants with Kaiser Permanente health plans. Shaded regions (10 April onwards) indicate admission dates when participants were excluded from analyses of clinical outcomes (duration of hospital stay, admission to intensive care unit, and case fatality risk) owing to limited follow-up duration 

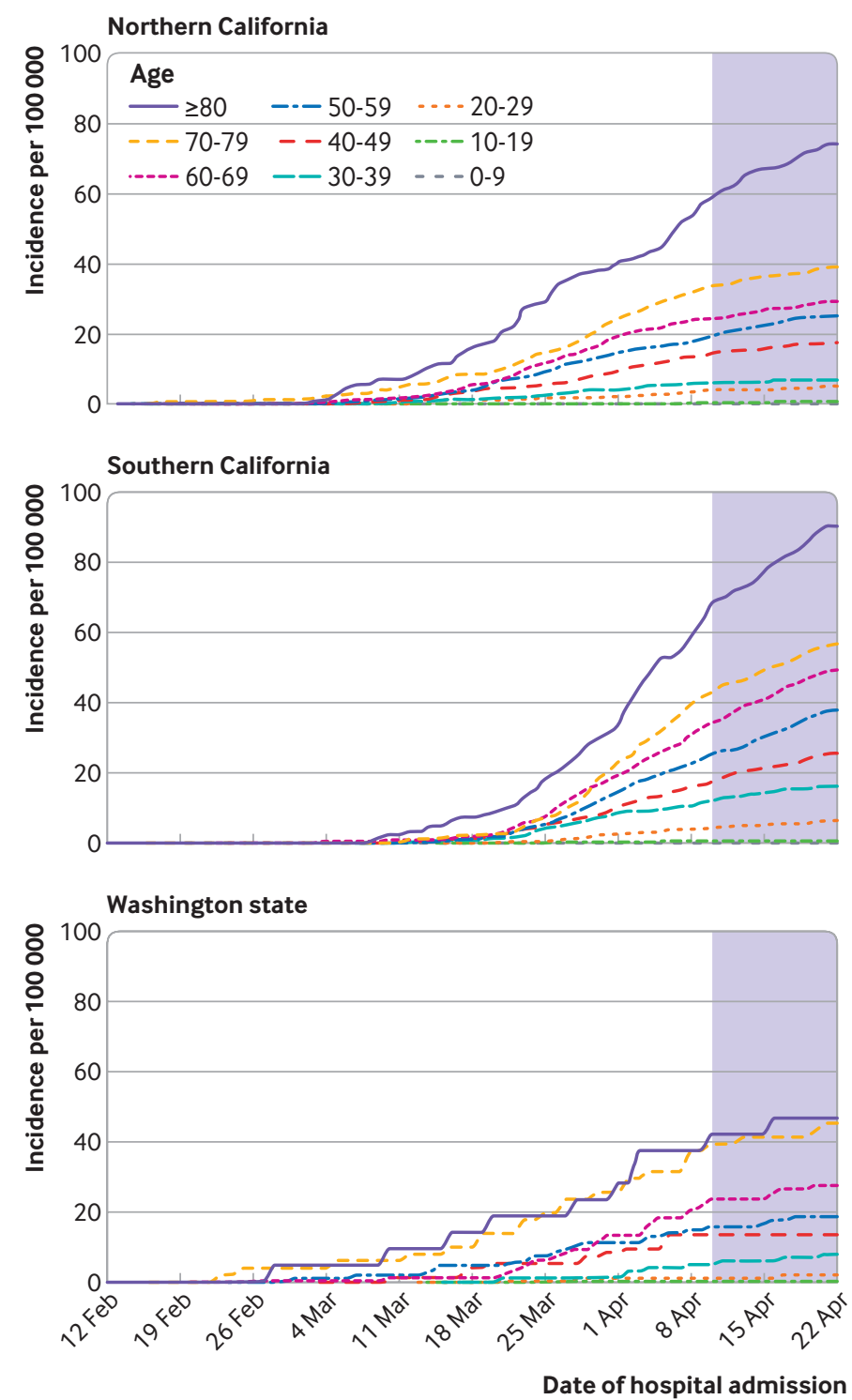

Fig 2 | Age specific cumulative incidence of first inpatient admissions in 2020 with confirmed coronavirus disease 2019 among participants with Kaiser Permanente health plans. Shaded regions (10 April onwards) indicate admission dates when participants were excluded from analyses of clinical outcomes (duration of hospital stay, admission to intensive care unit, and case fatality risk) owing to limited follow-up duration

\section{Discussion}

Our study provides an early assessment of the incidence and clinical profile of severe coronavirus disease 2019 (covid-19) among insured residents of California and Washington who receive comprehensive, integrated healthcare from Kaiser Permanente health plans. Incidence rates of hospital admissions for covid-19 increased within these cohorts from late February through mid-March 2020 and have since stabilized or declined amid implementation of social distancing measures. The highest rates of incidence occurred among older adults, with $50 \%$ of hospital admissions occurring among those aged 61 years and older and $25 \%$ among those aged 72 years and older. Consistent with differences in incidence of hospital admissions, the risk of death was higher in older patients. Long durations of hospital stay, in particular among non- survivors, indicate the potential for substantial healthcare burden associated with management of patients with severe covid-19. Our estimates also suggest that the effective reproduction number $\left(R_{E}\right)$ declined to a range near 1 after the implementation of social distancing interventions, concurrent with flattening of the incidence rate of new inpatient admissions for covid-19.

\section{Strengths and limitations of this study}

Our study has strengths as well as limitations. The Kaiser Permanente cohorts comprise a well monitored population with uniform standards of care and healthcare access, thus reducing the risk of ascertainment bias. Because healthcare capacity was not exceeded in the study regions and within Kaiser Permanente hospitals, hospital and ICU admission are more likely to reflect standard clinical practice during the study period compared with reports from other areas in which a surge in hospital admissions was considerably more pronounced. Although we did not conduct a detailed review of medical records for patients' presenting characteristics and associations with demographic factors, length of hospital stay, or clinical outcome, studies of clinical observations among patients in northern California ${ }^{30}$ and other parts of the US ${ }^{31}$ are now emerging and will facilitate comparison of infection severity and clinical outcomes across settings. Limited availability of laboratory testing in early phases of the US outbreak might have hindered detection of sporadic cases in January and early February 2020, as evidenced by the recent retrospective identification of a death from covid-19 in northern California in early February. ${ }^{32}$ Because all people under investigation for covid-19 might not have been tested during early phases of the outbreak, our estimates of disease incidence should be interpreted as lower bounds. Phylogenetic analyses of early SARSCoV-2 specimens will provide further insight into the extent of undetected transmission in the US, including in these Kaiser Permanente service regions. Last, sensitivity and specificity of SARS-CoV-2 laboratory tests used at facilities across these service regions might have differed.

In interpreting our estimates of $R_{E}$, it is important to consider that our analyses include hospital admissions among Kaiser Permanente enrollees only. Although those receiving healthcare from Kaiser Permanente generally resemble commercially insured populations of the same regions, ${ }^{89}$ they are likely to have higher socioeconomic status than those without insurance, and thus they do not reflect the general population. ${ }^{11}$ ${ }^{33}$ Our estimates of $R_{E}$ should therefore be taken to indicate transmission dynamics within communities and social networks within which socioeconomically vulnerable populations might be under-represented; nonetheless, pre-intervention and post-intervention estimates closely resemble state level estimates from other data sources. ${ }^{34}$ For analyses of transmission dynamics, we relied on data from other settings to infer parameters for the clinical course of covid-19, 

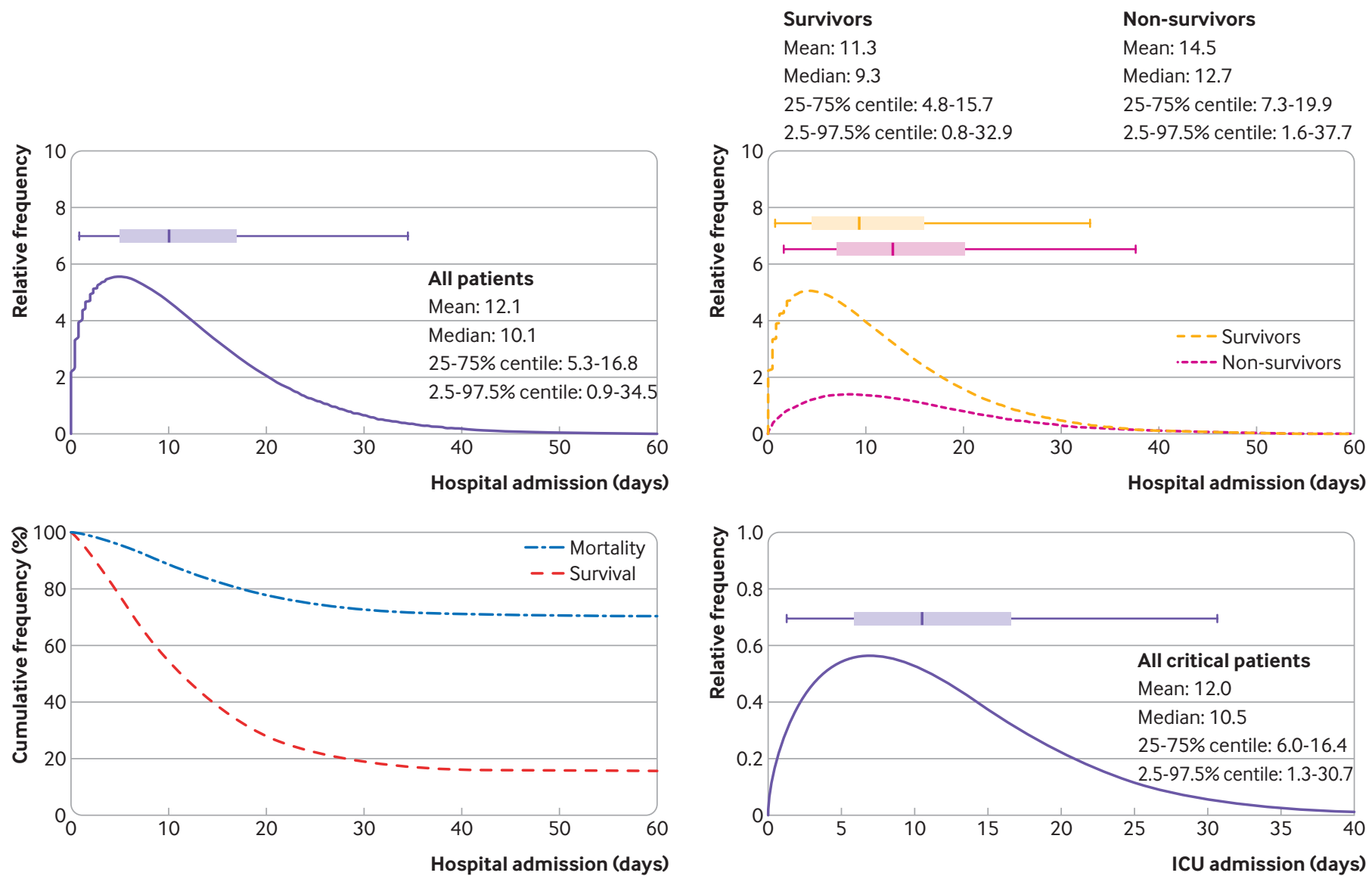

Fig 3 | Fitted durations of hospital admissions for first acute inpatient admissions with confirmed coronavirus disease 2019 by 9 April 2020 among all participants with Kaiser Permanente health plans admitted. Distributions of hospital length of stay for all acute inpatient admissions (top left); acute inpatient admissions, stratified by clinical outcome (top right); time to discharge or death among all acute inpatient admissions; and length of stay in an intensive care unit (ICU) for all inpatients admitted to ICU (bottom left). Box plots overlaid on density estimates indicate estimated medians (center line), interquartile ranges (boxed area), and $2.5 \%$ to $97.5 \%$ centile values (extended lines). Estimates were obtained by fitting Weibull distributed competing risk models to data on patient survival or non-survival as recorded by end of day 22 April 2020

including the proportion of people with symptoms requiring hospital admission, the serial interval, and the time from infection to hospital admission. Estimates of $R_{E}$ differed little across analyses using alternative parameterizations of these inputs, indicating our conclusions are robust to the underlying assumptions. Further, our use of data on people who were admitted to hospital in a prospectively followed cohort, receiving care within a unified healthcare delivery system, overcomes inconsistencies affecting studies of reported covid-19 cases or deaths across care providers and jurisdictions. ${ }^{35} 36$

\section{Comparison with other studies}

In many respects, our findings agree with observations in other settings, where older and male patients were more likely than younger or female patients to be admitted to the ICU and to die. ${ }^{237-39}$ The 11 day estimated mean duration of hospital stay for patients who are discharged alive is consistent with observations in China. ${ }^{37}$ However, we estimated a 15 day mean duration of stay among non-survivors, whereas in China non-survivors had a shorter length of hospital stay (7.5 days) than survivors. ${ }^{37}$ This difference might reflect, among other factors, alternative approaches to discharge and post-hospital or end-of-life care in diverse settings. These findings have important ramifications for anticipating clinical needs. A widely used model projecting clinical resource needs in the United Kingdom and US assumes a mean duration of hospital stay of eight days for most patients. ${ }^{6}$ Similarly, our finding that $40 \%$ of patients admitted to hospital received intensive care is higher than estimates used in numerous influential modeling studies. ${ }^{60-42}$

Our population of hospital patients was older on average than those admitted to hospital in China, ${ }^{23} 374344$ where the median age range was 5057 years. Notably, the median age of patients in our study matches reports from two case series in New York City. ${ }^{3145}$ This observation of older ages in US patients admitted to hospital might relate to underlying population demographic structure and differences in health status in US populations compared with those of other settings, or to differences across settings in the clinical threshold for hospital admission. A substantial proportion of the patients admitted to hospital and ICU were, however, younger or middle aged adults, in agreement with past reports that severe disease is not 


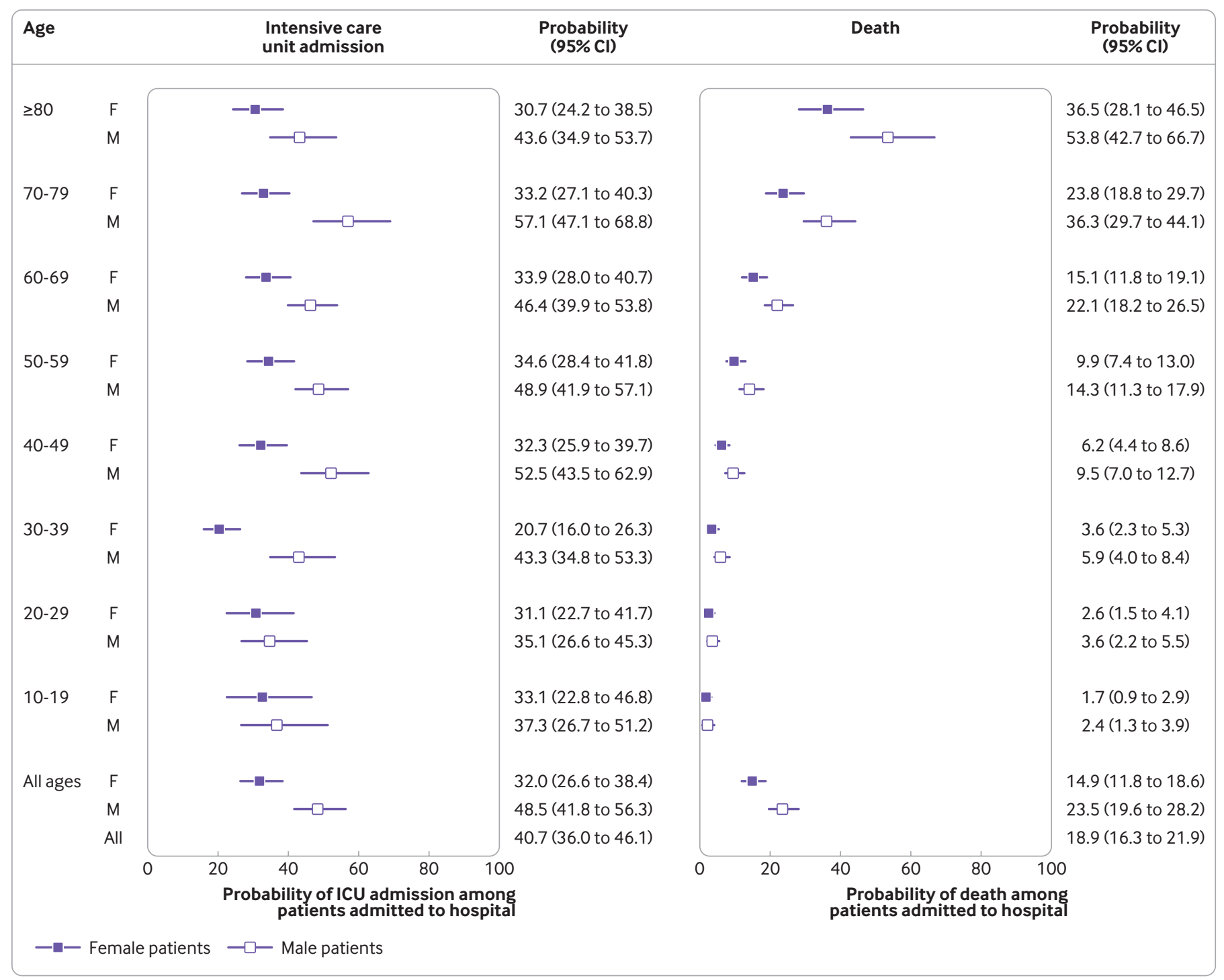

Fig 4 | Estimated probabilities of admission to intensive care unit (ICU) and mortality in enrollees of Kaiser Permanente health plans by age and sex for those with coronavirus disease 2019 (covid-19) admitted by 9 April 2020. Probabilities were stratified by age and sex for ICU admission (left) and mortality (right) for male and female patients within 10 year age stratums; children aged 0-9 years are excluded as none were admitted to hospital in this age group. Numerical estimates are indicated alongside plotted values. Sex specific estimates for all ages are plotted at the bottom. Estimates were obtained using parametric (Weibull) survival models to account for censoring of observations among incomplete hospital admissions

strictly limited to older patients. ${ }^{3845-47}$ Indeed, we did not identify strong evidence of a differential risk of ICU admission among hospital patients of differing ages.

\section{Conclusions and policy implications}

The considerable length of stay $(2.5-97.5 \%$ centile: 0.9-34.5) among patients admitted to hospital with covid-19 in our study indicates that unmitigated transmission of SARS-CoV-2 could threaten US hospital capacity, consistent with observations in Italy and other high resource settings ${ }^{3}$ as well as recent experience in New York City. ${ }^{2}$ Although current social distancing measures have provided a crucial stopgap in reducing transmission and protecting healthcare systems to date, ${ }^{48}$ hospitals should ensure capacity to manage patients with covid-19 in the coming months in a manner that is responsive to changes in social distancing or other pandemic mitigating measures.

\section{AUTHOR AFFILIATIONS}

${ }^{1}$ Division of Epidemiology and Biostatistics, School of Public Health, University of California, Berkeley, Berkeley, CA, 94720, USA

${ }^{2}$ Division of Infectious Diseases and Vaccinology, School of Public Health, University of California, Berkeley, Berkeley, CA, USA

${ }^{3}$ Center for Computational Biology, College of Engineering, University of California, Berkeley, Berkeley, CA, USA

${ }^{4}$ Division of Research, Kaiser Permanente, Oakland, CA, USA

${ }^{5}$ Health Research Institute, Kaiser Permanente Washington, Seattle, WA, USA

${ }^{6}$ Center for Health Research, Kaiser Permanente Northwest, Portland, OR, USA

${ }^{7}$ MRC Centre for Global Infectious Disease Analysis, Abdul Latif Jameel Institute for Disease and Emergency Analytics, Imperial College London, London, UK

${ }^{8}$ Department of Infectious Disease Epidemiology, Imperial College London, London, UK

${ }^{9}$ The Care Management Institute, Kaiser Permanente, Oakland, California 94612

${ }^{10}$ Department of Demography, University of California, Berkeley, Berkeley, CA, USA 
${ }^{11}$ Department of Medical Statistics, London School of Hygiene and Tropical Medicine, London, UK

${ }^{12}$ The Permanente Federation, Kaiser Permanente, Oakland, CA, USA

We thank for their role in organization of data capture and preparation for analyses: Alvina Sundang (The Permanente Federation, Kaiser Permanente); Jay Robles (The Care Management Institute, Kaiser Permanente); Lesley-Anne Myerscough, Yumi Wong, Gina Gourd Bademian (KP Insight Washington, Kaiser Permanente); and the nationwide clinical, administrative, and operational teams of Kaiser Permanente for their effort in pandemic response.

Contributors: JAL, VXL, MLJ, MAS, BLI, JPF, NPJ, SY, and JB conceived the study. JPF, CJ, and JB collected data. JAL, VXL, MLJ, MAS, BLJ, JPF, GRN, AM, NPJ, and JB designed the analyses. JAL, JPF, CJ, and GRN conducted the analyses. JAL generated the figure and wrote the first draft of the manuscript. All authors critically revised the manuscript for intellectual content. JAL acts as guarantor for this paper and accepts full responsibility for the work, had access to the data, and controlled the final decision to publish. The corresponding author attests that all listed authors meet authorship criteria and that no others meeting the criteria have been omitted.

Funding: This study was funded by Kaiser Permanente. VXL was supported by grant R35GM128672 from the US National Institutes of Health. VXL, MLI, MAS, JPF, CI, SY, and IB are employees of Kaiser Permanente; however, Kaiser Permanente officers and employees other than the authors did not play a role in study design; in the collection, analysis, and interpretation of data; in the writing of the report; or in the decision to submit the article for publication. All authors had full access to all of the data (including statistical reports and tables) in the study and can take responsibility for the integrity of the data and the accuracy of the data analysis.

Competing interests: All authors have completed the ICMJE uniform disclosure form at www.icmje.org/coi_disclosure.pdf. JAL, NPJ, and BLJ have received honorariums from Kaiser Permanente. VXL was supported by grant R35GM128672 from the US National Institutes of Health. All other authors declare: no support from any organization for the submitted work; no financial relationships with any organizations that might have an interest in the submitted work in the previous three years; no other relationships or activities that could appear to have influenced the submitted work.

Ethical approval: Retrospective reviews of deidentified data for this study were considered exempt, non-human subjects research by the Kaiser Permanente institutional review boards for northern California, southern California, and Washington state.

Data sharing: The supplementary tables provide aggregate data to reproduce the analyses.

Dissemination to participants and related patient and public communities: Results of this study have been made available to the public through an open access preprint posted to MedRxiv (doi:10.1 $101 / 2020.04 .12 .20062943)$ and have been communicated directly to policy makers. A lay version of the results will be disseminated to Kaiser Permanente healthcare plan members and the public by the Kaiser Permanente press office.

The manuscript's guarantor (JAL) affirms that the manuscript is an honest, accurate, and transparent account of the study being reported; that no important aspects of the study have been omitted; and that any discrepancies from the study as originally planned have been explained.

This is an Open Access article distributed in accordance with the Creative Commons Attribution Non Commercial (CC BY-NC 4.0) license, which permits others to distribute, remix, adapt, build upon this work non-commercially, and license their derivative works on different terms, provided the original work is properly cited and the use is noncommercial. See: http://creativecommons.org/licenses/by-nc/4.0/.

1 World Health Organization. Coronavirus disease 2019 (COVID-19) Situation Report - 102. 2020. https://www.who.int/emergencies/ diseases/novel-coronavirus-2019/situation-reports. Accessed May 1,2020

2 Centers for Disease Control and Prevention. Coronavirus disease 2019 (COVID-19) cases in US. https://www.cdc.gov/coronavirus/2019ncov/cases-updates/cases-in-us.html. Accessed May 1, 2020.

3 Remuzzi A, Remuzzi G. COVID-19 and Italy: what next?Lancet 2020;395:1225-8. doi:10.1016/S01406736(20)30627-9

4 Tanne JH, Hayasaki E, Zastrow M, Pulla P, Smith P, Rada AG. Covid-19: how doctors and healthcare systems are tackling coronavirus worldwide. BMJ 2020;368:m1090. doi:10.1136/bmj.m1090
5 Khan S, Nabi G, Han G, et al. Novel coronavirus: how things are in Wuhan. Clin Microbiol Infect 2020;26:399-400. doi:10.1016/j. cmi.2020.02.005

6 Ferguson NM, Laydon D, Nedjati-Gilani G, et al. Impact of non-pharmaceutical interventions (NPIs) to reduce COVID-19 mortality and healthcare demand. Imperial College, 2020, doi: $10.25561 / 77482$

7 Chowell G, Mizumoto K. The COVID-19 pandemic in the USA: what might we expect? Lancet 2020;395:1093-4. doi:10.1016/S01406736(20)30743-1

8 Koebnick C, Langer-Gould AM, Gould MK, et al. Sociodemographic characteristics of members of a large, integrated health care system: comparison with US Census Bureau data. Perm J 2012;16:37-41. doi:10.7812/TPP/12-031

9 Gordon NP. Similarity of the adult Kaiser Permanente membership in Northern California to the insured and general population in Northern California: Statistics from the 2011 California Health Interview Survey. https://divisionofresearch.kaiserpermanente.org/projects/ memberhealthsurvey/SiteCollectionDocuments/chis_non_kp_2011. pdf. Accessed May 1, 2020

10 Saunders KW, Davis RL, Stergachis A. Group health cooperative. Pharmacoepidemiology, 2006: 223-39.

11 Stiefel MC, Gordon NP, Wilson-Anumudu FJ, Arsen EL. Sociodemographic determinants of health and well-being among adults residing in the combined Kaiser Permanente regions. Perm 12019:23:18-091. doi:10.7812/TPP/18-091

12 Kaiser Permanente Geographically Enriched Member Sociodemographics Datamart (GEMS). Accessed April 30, 2020.

13 Ryan CL, Bauman K. Educational attainment in the United States: 2015. Curr Popul Rep [Spec Censuses] 2016.

14 Kaiser Permanente Emergency Management. COVID-19 intensive care unit, medical surgical and medical surgical telemetry unit surge playbook. 2020. https://permanente.org/wp-content/ uploads/2020/03/KP-NCAL-COVID-MST-Playbook_March-30.pdf. Accessed May 1, 2020

15 Centers for Disease Control and Prevention. Interim Guidance: Discontinuation of transmission-based precautions and disposition of patients with COVID-19 in healthcare settings. 2020. https:// www.cdc.gov/coronavirus/2019-ncov/hcp/disposition-hospitalizedpatients.html. Accessed May 1, 2020.

16 Mahani AS, Sharabiani MTA. Bayesian, and non-bayesian, causespecific competing-risk analysis for parametric and nonparametric survival functions: The R Package CFC. J Stat Softw 2019:89:(9). doi:10.18637/jss.v089.109

17 Wallinga J, Teunis P. Different epidemic curves for severe acute respiratory syndrome reveal similar impacts of control measures. Am J Epidemiol 2004;160:509-16. doi:10.1093/aje/kwh255

18 Tindale L, Coombe M, Stockdale JE, et al. Transmission interval estimates suggest pre-symptomatic spread of COVID-19. MedRxiv 20029983 [Preprint]. 2020. doi:10.1101/2020.03.03.20029983

19 Backer JA, Klinkenberg D, Wallinga J. Incubation period of 2019 novel coronavirus (2019-nCoV) infections among travellers from Wuhan, China, 20-28 January 2020. Euro Surveill 2020;25. doi:10.2807/1560-7917.ES.2020.25.5.2000062

20 Lauer SA, Grantz KH, Bi Q et al. The incubation period of coronavirus disease 2019 (COVID-19) from publicly reported confirmed cases: estimation and application. Ann Intern Med 2020;172:577-82. doi:10.7326/M20-0504

21 Linton NM, Kobayashi T, Yang Y, et al. Incubation period and other epidemiological characteristics of 2019 novel coronavirus infections with right truncation: a statistical analysis of publicly available case data. / Clin Med 2020;9:E538. doi:10.3390/ jcm9020538

22 Zhang J, Litvinova M, Wang W, et al. Evolving epidemiology and transmission dynamics of coronavirus disease 2019 outside Hubei province, China: a descriptive and modelling study. Lancet Infect Dis 2020; published online 2 April. doi:10.1016/S14733099(20)30230-9

23 Wang D, Hu B, Hu C, et al. Clinical characteristics of 138 hospitalized patients with 2019 novel coronavirus-infected pneumonia in Wuhan, China. JAMA 2020; published online 7 February. doi:10.1001/ jama.2020.1585

24 Verity R, Okell LC, Dorigatti I, et al. Estimates of the severity of coronavirus disease 2019: a model-based analysis. Lancet Infect Dis 2020:3099:1-9. doi:10.1016/S1473-3099(20)30243-7

25 Salje H, Tran Kiem C, Lefrancq N, et al. Estimating the burden of SARS-CoV-2 in France. MedRxiv 20072413 [Preprint]. 2020. doi:10.1101/2020.04.20.20072413

26 Du Z, Xu X, Wu Y, Wang L, Cowling BJ, Meyers LA. Serial interval of COVID-19 among publicly reported confirmed cases. Emerg Infect Dis 2020;26. doi:10.3201/eid2606.200357

27 Nishiura H, Linton NM, Akhmetzhanov AR. Serial interval of novel coronavirus (COVID-19) infections. Int I Infect Dis 2020;93:284-6. doi:10.1016/j.jij. 2020.02.060 
28 Li Q, Guan X, Wu P, et al. Early transmission dynamics in Wuhan, China, of novel coronavirus-Infected pneumonia. N Engl) Med 2020;382:1199-207. doi:10.1056/NEJMoa2001316

29 Lasry A, Kidder D, Hast M, et al. CDC Public Health Law Program, New York City Department of Health and Mental Hygiene, Louisiana Department of Health, Public Health - Seattle \& King County, San Francisco COVID-19 Response Team, Alameda County Public Health Department, San Mateo County Health Department, Marin County Division of Public Health. Timing of community mitigation and changes in reported COVID-19 and community mobility - four U.S. metropolitan areas, February 26-April 1, 2020. MMWR Morb Mortal Wkly Rep 2020;69:451-7. doi:10.15585/mmwr.mm6915e2

30 Myers LC, Parodi SM, Escobar GJ, Liu VX. Characteristics of hospitalized adults with COVID-19 in an integrated health care system in California. JAMA 2020;40:24-6. doi:10.1001/ jama.2020.7202

31 Petrilli CM, Jones SA, Yang J, et al. Factors associated with hospitalization and critical illness among 4,103 patients with COVID-19 disease in New York City. MedRxiv 20057794 [Preprint] 2020. doi:10.1101/2020.04.08.20057794

32 Fuller T, Baker M, Hubler S, et al. A coronavirus death in early February was 'probably the tip of an iceberg'. New York Times. 2020. https://www.nytimes.com/2020/04/22/us/santa-clara-countycoronavirus-death.html. Accessed May 1, 2020.

33 Gordon N, Lin T. The Kaiser Permanente Northern California Adult Member Health Survey. Perm / 2016;20:15-225. doi:10.7812/ TPP/15-225

34 Center for Mathematical Modelling of Infectious Diseases, London School of Hygiene and Tropical Medicine. Temporal variation in transmission during the COVID-19 outbreak: National and subnational estimates for the United States of America. 2020. https://epiforecasts.io/covid/. Accessed May 1, 2020.

35 Jewell NP, Lewnard JA, Jewell BL. Predictive mathematical models of the COVID-19 pandemic: Underlying principles and value of projections. JAMA 2020; published online 16 April. doi:10.1001/ jama.2020.6585

36 Jewell NP, Lewnard JA, Jewell BL. Caution warranted: Using the Institute for Health Metrics and Evaluation model for predicting the course of the COVID-19 pandemic. Ann Intern Med 2020; published online 14 April. doi:10.7326/M20-1565

37 Zhou F, Yu T, Du R, et al. Clinical course and risk factors for mortality of adult inpatients with COVID-19 in Wuhan, China: a retrospective cohort study. Lancet 2020;395:1054-62. doi:10.1016/S01406736(20)30566-3

38 Grasselli G, Zangrillo A, Zanella A, et al, COVID-19 Lombardy ICU Network. Baseline characteristics and outcomes of 1591 patients infected with SARS-CoV-2 admitted to ICUs of the Lombardy Region, Italy. JAMA 2020; published online 6 April. doi:10.1001/ jama.2020.5394

39 Onder G, Rezza G, Brusaferro S. Case-fatality rate and characteristics of patients dying in relation to COVID-19 in Italy. JAMA 2020; published online 23 March. doi:10.1001/jama.2020.4683

40 Li R, Rivers C, Tan Q, et al. The demand for inpatient and ICU beds for COVID-19 in the US: lessons from Chinese cities. MedRxiv 20033241 [Preprint]. 2020. doi:10.1101/2020.03.09.20033241

41 Jit M, Jombart T, Nightingale E, et al. Estimating number of cases and spread of coronavirus disease 2019 (COVID-19) in the United Kingdom using critical care admissions, February to March 2020. MedRxiv 20054528 [Preprint]. 2020. doi:10.1101/2020.04.05.20054528

42 Tuite AR, Fisman DN, Greer AL. Mathematical modelling of COVID-19 transmission and mitigation strategies in the population of Ontario, Canada. CMA/ 2020; published online 8 April. doi:10.1503/ cmaj.200476

43 Qian G-Q Yang N-B, Ding F et al. Epidemiologic and Clinical Characteristics of 91 Hospitalized Patients with COVID-19 in Zhejiang, China: A retrospective, multi-centre case series. QJM 2020; published online 17 March. doi:10.1093/qjmed/hcaa089

44 Liu K, Fang Y-Y, Deng Y, et al. Clinical characteristics of novel coronavirus cases in tertiary hospitals in Hubei Province. Chin Med J (Engl) 2020;133:1025-31. doi:10.1097/ CM9.0000000000000744

45 Richardson S, Hirsch JS, Narasimhan M, et al, and the Northwell COVID-19 Research Consortium. Presenting characteristics, comorbidities, and outcomes among 5700 patients hospitalized with COVID-19 in the New York City Area. JAMA 2020; published online 22 April. doi:10.1001/jama.2020.6775

46 Guan WJ, Ni ZY, Hu Y, et al, China Medical Treatment Expert Group for Covid-19. Clinical characteristics of coronavirus disease 2019 in China. N Engl / Med 2020;382:1708-20. doi:10.1056/ NEJMoa2002032

47 Wu Z, McGoogan JM. Characteristics of and important lessons from the coronavirus disease 2019 (COVID-19) outbreak in China. JAMA 2020; published online 24 February. doi:10.1001/ jama.2020.2648

48 Lewnard JA, Lo NC. Scientific and ethical basis for social-distancing interventions against COVID-19. Lancet Infect Dis 2020; published online 23 March doi:10.1016/S1473-3099(20)30190-0

Supplementary information: additional tables and figures 